\title{
BMJ Open Functional health literacy and glycaemic control in older adults with type 2 diabetes: a cross-sectional study
}

\author{
Jonas Gordilho Souza, Daniel Apolinario, Regina Miksian Magaldi, \\ Alexandre Leopold Busse, Flavia Campora, Wilson Jacob-Filho
}

To cite: Souza JG, Apolinario D, Magaldi RM, et al. Functional health literacy and glycaemic control in older adults with type 2 diabetes: a cross-sectional study. BMJ Open 2014;4: e004180. doi:10.1136/ bmjopen-2013-004180

- Prepublication history and additional material for this paper is available online. To view these files please visit the journal online (http://dx.doi.org/10.1136/ bmjopen-2013-004180).

Received 6 October 2013 Revised 16 January 2014 Accepted 21 January 2014

\section{CrossMark}

Geriatrics Division, Department of Internal Medicine, University of São Paulo Medical School, São Paulo, Brazil

Correspondence to Dr Daniel Apolinario; daniel.apolinario@usp.br

\author{
ABSTRACT \\ Objectives: To investigate the relationship between \\ functional health literacy and glycaemic control in a \\ sample of older patients with type 2 diabetes. \\ Design: Cross-sectional study. \\ Setting: A government-financed outpatient geriatric \\ clinic in São Paulo, Brazil.
}

Participants: 129 older patients with type 2 diabetes, a mean (SD) age of 75.9 (6.2) years, a mean glycosylated haemoglobin (HbA1c) of $7.2 \%(1.4)$, of which $14.7 \%$ had no formal education and $82.9 \%$ had less than a high-school diploma.

Measures: HbA1c was used as a measure of glycaemic control. Functional health literacy was assessed with the 18-item Short Assessment of Health Literacy for Portuguese-speaking Adults (SAHLPA-18), a validated instrument to evaluate pronunciation and comprehension of commonly used medical terms. Regression models were controlled for demographic data, depressive symptoms, diabetes duration, treatment regimen, diabetes knowledge and assistance for taking medications.

Results: Functional health literacy below adequate was encountered in $56.6 \%$ of the sample. After controlling for potential confounding factors, patients with inadequate functional health literacy were more likely than patients with adequate functional health literacy to present poor glycaemic control $(\mathrm{OR}=4.76 ; 95 \% \mathrm{Cl} 1.36$ to 16.63). In a fully adjusted linear regression model, lower functional health literacy $(\beta=-0.42 ; p<0.001)$, longer diabetes duration $(\beta=0.24 ; p=0.012)$ and lack of assistance for taking medications $(\beta=0.23 ; p=0.014)$ were associated with higher levels of $\mathrm{HbA} 1 \mathrm{c}$. Contrary to our expectations, illiterate patients did not have poorer outcomes when compared with patients with adequate functional health literacy, raising the hypothesis that illiterate individuals are more likely to have their difficulties recognised and compensated. However, the small subsample of illiterate patients provided limited power to reject differences with small magnitude.

Conclusions: Patients with inadequate functional health literacy presented with higher odds of poor glycaemic control. These findings reinforce the importance of addressing limited functional health literacy in clinical practice.

\section{Strengths and limitations of this study}

- The study included a sample which represents the lowest levels of the health literacy spectrum, where the association of health literacy with health outcomes is deemed to be stronger.

- A wide range of potentially confounding variables has been controlled and we have made a careful selection of the sample, excluding conditions that can affect the accuracy of the HbA1C assay or determine different targets of glycaemic control.

- The study was conducted in a governmentfinanced health system which provides medications at no cost. This factor may attenuate inequalities in access to therapeutic resources, making the role of functional health literacy more evident.

- Although we have excluded individuals with a clinical diagnosis of dementia, we did not screen for dementia and did not make adjustments for cognitive performance.

- The relatively small subsample of illiterate patients provided limited power to reject risk differences with small magnitude.

\section{INTRODUCTION}

Health literacy has been defined by the WHO as 'the cognitive and social skills which determine the motivation and ability of individuals to gain access to, understand and use information in ways which promote and maintain good health'. ${ }^{1}$ The concept of health literacy is not restricted to the ability to read and follow medical instructions. It includes a range of communicative and critical skills such as searching for specific health knowledge, evaluating information for credibility, balancing risks and benefits, expressing needs and negotiating preferences.

The term 'functional health literacy' has been used to imply one's ability to function adequately in healthcare settings, as determined by instruments which access basic skills 
needed to deal with health-related written materials. ${ }^{2}$ This somewhat narrow approach misses the richness implied by the WHO definition, but warrants practical feasibility for studies investigating the relationships between health literacy and health outcomes. Inadequate functional health literacy has been independently associated with poorer ability to take medications appropriately, lower utilisation of preventive services, more hospitalisations, poorer overall health status and higher mortality rates. ${ }^{3}$ Multiple interventions exist to lessen the negative effects of inadequate functional health literacy, ${ }^{4}$ but health professionals often overestimate patients' abilities and fail to recognise the problem. $^{5}$

Some segments of the population are at greater risk for inadequate health literacy, including the socioeconomically disadvantaged, immigrants and older adults. In a study that included Medicare enrollees aged 65 years and older, functional health literacy skills declined dramatically with age, even after adjusting for educational status and cognitive impairment. ${ }^{6}$ Inadequate health literacy may disproportionately affect the health of older persons, not only because it is more prevalent in this age group but also because older persons are more exposed to healthcare services and more likely to receive complex therapeutic regimens. ${ }^{7}$

Diabetes care involves extensive self-management behaviours and requires pharmacological regimens that tend to become increasingly complex over time. This prototypical chronic disease constitutes a representative model for studying the influence of health literacy on health outcomes. However, although many theoretical mechanisms are proposed that link health literacy to diabetes self-care and clinical outcomes, the direct association between functional health literacy and glycaemic control is still controversial. ${ }^{8}$

In a recent systematic review, 13 studies were included that have explored the relationship between health literacy and glycaemic control. ${ }^{9}$ Results were inconsistent across studies, and the heterogeneity did not allow the estimation of an overall effect. Therefore, the evidence for direct association was rated insufficient. These findings may indicate that health literacy is related to certain outcomes in particular populations, but not in others. Only one study was conducted in a developing country, including a population with very low levels of educational attainment. ${ }^{10}$ In that study, higher scores on the Short Test of Functional Health Literacy in Adults (S-TOFHLA) were strongly associated with reduced levels of glycosylated haemoglobin (HbA1c). In general, there is paucity of data on the association between health literacy and glycaemic control in patients who have only rudimentary reading skills and in those who are unable to read at all.

The purpose of this study was to determine the independent association of functional health literacy with glycaemic control in a Brazilian sample of heterogeneous, predominantly low-educated older adults with type 2 diabetes.

\section{METHODS}

\section{Subjects}

A convenient sample was recruited between June 2011 and July 2012 from a government-financed outpatient geriatric clinic in the city of São Paulo, southeastern Brazil. During this period, 225 older adults with type 2 diabetes were screened for participation. At the time of the study, there was no diabetes management programme or educational intervention in place. All patients were treated by geriatricians or geriatricians in training, were provided diabetes medications at no cost and had access to the same range of services.

Research staff reviewed medical records and spoke with patients to verify inclusion and exclusion criteria. Eligibility criteria included: (1) age $\geq 60$ years; (2) selfreported ability to speak fluent Portuguese; (3) type 2 diabetes currently being treated with daily medication and (4) an HbAlc measurement performed within a 6-month period. Patients were ineligible if they had a hearing, vision, motor or speech problem that precluded adequate interaction with the interviewer or impeded appropriate completion of the proposed assessments. Patients with a diagnosis of dementia were excluded because cognitive impairment has been associated with poor performance in functional health literacy tests and may affect an individual's ability to manage drug regimens. ${ }^{11} 12$ Patients with overt thyroid dysfunction (thyroid-stimulating hormone $<0.1$ or $>10 \mathrm{mU} / \mathrm{L}$ ), anaemia (haemoglobin $<11 \mathrm{mg} / \mathrm{dL}$ for men and $<10 \mathrm{mg} / \mathrm{dL}$ for women) and severe renal failure (estimated glomerular filtration rate $<30 \mathrm{~mL} / \mathrm{min} / 1.73 \mathrm{~m}^{2}$ ) were excluded because these conditions can affect the accuracy of the HbAlc assay. ${ }^{13-15}$

We further excluded participants who fulfilled criteria for frailty, because less stringent targets of glycaemic control have been proposed for the frail elderly. ${ }^{16}$ Frailty status was determined according to the Study of Osteoporotic Fracture (SOF) index. ${ }^{17}$ The SOF index is composed of the following three items: (1) weight loss of more than $5 \%$ during the last year; (2) inability to rise from a chair five consecutive times without using the arms and (3) self-perceived reduced energy level. Participants were assessed systematically and excluded if at least two of the three criteria were fulfilled.

Eligible patients attending scheduled appointments were approached in the clinic waiting room and provided a description of the study procedures. Informed consent was obtained before the interview. The consent form was read aloud and explained in plain language for those individuals who declared being illiterate or were judged by the interviewer as having questionable capacity to understand the form.

\section{Demographic and clinical data}

All participants were interviewed for demographic information, including age, gender, educational attainment (highest grade completed), race (white or non-white) and lifetime occupation (predominantly manual or non- 
manual). Individuals were further classified as married (including cohabiting) or unmarried (never married, divorced or widowed). Economic status was determined according to the Brazilian Economic Classification Criterion, ${ }^{18}$ which provides a discrete scale calculated by assigning scores to the number of household assets.

Duration of diabetes was registered and treatment was characterised as oral agents alone or an insulincontaining regimen. Participants were further asked if they had supervision or help in taking medications and classified as receiving assistance or not. As some studies have reported depression as an important factor influencing glycaemic control, ${ }^{19}$ we assessed depressive symptoms using the 15-Item Geriatric Depression Scale (GDS-15)..$^{20} 21$

\section{Functional health literacy}

We assessed functional health literacy by using the 18-item Short Assessment of Health Literacy for Portuguese-speaking Adults (SAHLPA-18), a test that evaluates pronunciation and comprehension of commonly used medical terms. SAHLPA-18 has been previously validated in a sample of Brazilian older adults, presenting moderate-to-high correlations with construct criteria, high internal consistency and adequate testretest reliability. ${ }^{22}$

We used laminated flash cards, each with a medical term printed in boldface on the top and two association words at the bottom. One of the words is meaningfully associated with the medical term and the other is not. Respondents were shown flash cards one at a time and asked to read aloud the medical term in boldface. The interviewer then read the two association words and asked which one was meaningfully associated with the medical term. As the purpose of the association questions was to assess comprehension, respondents were instructed not to guess and say 'don't know' if they did not know the correct association. The answer was deemed correct only when the respondent correctly pronounced the medical term and made the correct association. One point was scored for each correct item with a maximum score of 18 . Using previously validated criteria, $^{22}$ we categorised patients as having inadequate functional health literacy if the SAHLPA-18 score was 1-14 and adequate functional health literacy if it was 15-18. Patients were considered illiterates if the SAHLPA-18 score was 0 or if individuals did not attempt to complete the test alleging being unable to read at all.

\section{Diabetes knowledge}

Diabetes knowledge was assessed with the Spoken Knowledge in Low Literacy patients with Diabetes (SKILLD), a 10-item test with questions about behaviours patients should have to best manage their diabetes. ${ }^{23}$ SKILLD is verbally administrated-questions are read aloud in an open-ended format and answers are recorded as either correct or incorrect. Full marks are given only for complete answers and all questions are weighted equally. SKILLD was chosen because it provides measures of diabetes knowledge that are more independent of the literacy status, as it does not require reading tasks and the patients are allowed to explain answers in their own words.

\section{Diabetes outcomes}

The most recent $\mathrm{HbAlc}$ value extracted from the electronic medical record was used as a measure of glycaemic control, reflecting the mean glycaemia over the preceding 2-3 months. Recently published guidelines from the American Geriatrics Society Expert Panel on the Care of Older Adults with Diabetes Mellitus recommend that the 'target goal for HbAlc in older adults generally should be 7.5-8\%'.16 Accordingly, inadequate glycaemic control was defined as $\mathrm{HbA1c}>8 \%$. Data on diabetes complications (ie, retinopathy, nephropathy and neuropathy) were obtained from medical records and registered as dichotomous variables (present or absent).

\section{Statistical analysis}

We performed descriptive statistics to characterise the sample and the studied variables. Patients were classified according to their functional health literacy status in one of three categories: illiteracy, inadequate functional health literacy or adequate functional health literacy. Variables were compared between the three groups using the Fisher exact test for categorical data, one-way analyses of variance (ANOVA) for continuous parametric data and Kruskal-Wallis for continuous non-parametric data. Post hoc tests for determining differences between means were accomplished with Tukey's honestly significant difference procedure. Effect sizes were calculated using Cohen's d method.

Regression analyses were used to explore the association between functional health literacy and glycaemic control while controlling for other potentially confounding variables. In primary analyses, functional health literacy and glycaemic control were used as continuous variables in hierarchical multiple linear regressions. In these models, HbAlc was the dependent variable and SAHLPA-18 was the primary independent variable. Covariates were entered in four sequential steps for examining their incremental validity, as indicated by changes in the coefficient of determination $\left(R^{2}\right)$. In the first step, SAHLPA-18 was entered without covariates. In the second step, seven sociodemographic variables were entered as a block (ie, age, gender, race, educational attainment, occupation, economic status and marital status). The incremental role of four clinical variables was examined in the third step (ie, depressive symptoms, diabetes duration, treatment regimen and assistance for taking medications). SKILLD was entered as the last step, because diabetes knowledge represents a further adjustment factor deemed to be interrelated with health literacy and that has not been used as a covariate in many studies. As SAHLPA-18 does not provide useful 
measures in illiterates, those individuals were not included in multiple linear models. Moreover, as regression models have included a substantial number of correlated variables, multicollinearity was assessed using the variance inflation factor (VIF).

In secondary analyses, functional health literacy and glycaemic control were taken as categorical variables in logistic regression models. The odds of inadequate glycaemic control were calculated separately for patients with illiteracy and inadequate health literacy taking patients with adequate functional health literacy as a reference. We also used logistic regression models to determine the independent effect of health literacy on the risk of diabetes complications.

Two-way interactions were carried out to investigate whether the impact of functional health literacy on glycaemic control differs according to age, gender, depressive symptoms, diabetes duration, insulin use and assistance for taking medications. In addition, we have investigated interactions between all variables that were significantly associated with glycaemic control in the fully adjusted linear regression model.

Based on a two-sided significance level of 0.05 and $80 \%$ power, we estimated a total sample size of 128 patients for detecting differences between two groups with medium effect sizes (Cohen's $d=0.5$ ). Statistical analyses were performed with Stata V.12.1 (Stata Corp. LP, College Station, Texas, USA) and power calculations were conducted with the software $G^{*}$ Power 3.1.5. ${ }^{24}$ All statistical tests were two-tailed, and an $\alpha$ level of less than 0.05 was used to indicate statistical significance.

\section{RESULTS}

Two hundred and twenty-five older adults with type 2 diabetes were screened for participation. Of these, 66 were ineligible because they had dementia $(n=51)$, renal failure $(n=9)$, did not speak fluent Portuguese $(n=3)$ and had anaemia $(n=2)$ and aphasia $(n=1)$. All the remaining 159 patients were approached at a clinical appointment. Of these, 13 were excluded because they were considered frail according to the SOF criteria, 10 presented with poor visual acuity, 1 had severe hearing impairment and 6 refused to participate.

Our sample consisted of 129 older adults with a mean age (SD) of 75.9 (6.2) years, $69.8 \%$ of whom were women. Median educational attainment was 4 years, with $14.7 \%$ of the individuals having no formal education and $82.9 \%$ having less than a high-school diploma. Functional health literacy below adequate as measured by SAHLPA- 18 was encountered in $56.6 \%$ of the sample $-11.6 \%$ who were illiterate and $45 \%$ who presented with inadequate functional health literacy. Overall, the median duration since the diagnosis of diabetes was 10 years, $31.8 \%$ of the patients were taking insulin and the mean (SD) HbAlc was $7.2 \%$ (1.4). Based on the most recent results for $\mathrm{HbA1c}, 52.7 \%$ of the patients were in tight control (HbAlc $\leq 7 \%), 24.8 \%$ were in fair control (HbAlc 7.1-8\%) and 22.5\% presented with inadequate glycaemic control (HbAlc >8\%). Table 1 lists additional demographic and clinical characteristics of the sample.

Patients with lower functional health literacy levels were more likely to have a non-white ethnicity, present a lower economic status, have a manual occupation, report a lower educational attainment and have less diabetes-specific knowledge. Overall, one-way ANOVA detected a significant difference in HbAlc means across functional health literacy levels $(p=0.034)$. Post hoc tests revealed a significant difference between individuals with adequate and inadequate functional health literacy (HbAlc 6.96 vs 7.56; $p=0.049$ ), with a Cohen's d of 0.44 indicating a medium effect size. ${ }^{25}$ There was no difference in glycaemic control between individuals with adequate functional health literacy and those who were considered illiterates (HbAlc 6.96 vs 6.85 ; $\mathrm{p}=0.953$ ).

In linear regression models, lower SAHLPA-18 scores were associated with higher levels of HbA1c throughout all adjustment steps. In the fully adjusted model, SAHLPA-18 was the variable more strongly associated with glycaemic control, with a standardised $\beta$ of $-0.42(\mathrm{p}<0.001)$. This means that, with all other variables held constant, a $1 \mathrm{SD}$ increase on SAHLPA-18 would be associated with an improvement of $0.42 \mathrm{SD}$ on the predicted HbAlc. The other variables associated with a poorer glycaemic control in the fully adjusted model were lack of assistance for taking medications and longer diabetes duration (table 2). The maximum VIF was 2.11 and mean VIF was 1.46, indicating that multicollinearity was not a problem. Interaction analyses did not yield any significant effects.

In fully adjusted logistic regression models, patients with inadequate functional health literacy were more likely than patients with adequate functional health literacy to present with inadequate glycaemic control (HbAlc $>8 \%)$ and with adjusted OR of 4.76 (95\% CI 1.36 to 16.63$)$. We did not find significant associations between inadequate functional health literacy and diabetes complications. In all adjusted models, illiterate individuals did not have poorer diabetes outcomes when compared with individuals with adequate functional health literacy (table 3).

\section{DISCUSSION}

In a sample of low-educated older patients with type 2 diabetes, our study demonstrates that lower scores on a functional health literacy test are associated with a higher likelihood of poor glycaemic control after adjusting for potential confounders. Our result is consistent with that reported by Tang et al, ${ }^{10}$ who studied a sample of Chinese patients with educational levels that are compatible with those encountered in our sample.

It has been suggested that there may be a threshold for the association between health literacy and health outcomes, that is, a certain level of health literacy is needed for a good outcome, but higher levels add little 
Table 1 Characteristics of the sample stratified by the functional health literacy level

\begin{tabular}{|c|c|c|c|c|c|}
\hline \multirow[b]{2}{*}{ Characteristics } & \multirow[b]{2}{*}{ Total $(n=129)$} & \multicolumn{3}{|c|}{ Functional health literacy level } & \multirow[b]{2}{*}{ p Value } \\
\hline & & $\begin{array}{l}\text { Illiterate } \\
(n=15)\end{array}$ & $\begin{array}{l}\text { Inadequate } \\
(n=58)\end{array}$ & $\begin{array}{l}\text { Adequate } \\
(n=56)\end{array}$ & \\
\hline Age, mean (SD), years & $75.9(6.2)$ & $78.2(6.3)$ & $75.9(5.9)$ & $75.3(6.4)$ & 0.280 \\
\hline Female gender, No. (\%) & $90(69.8)$ & $14(93.3)$ & $37(63.8)$ & 39 (69.6) & 0.074 \\
\hline White race, No. (\%) & 61 (47.9) & $4(26.7)$ & $21(36.2)$ & $36(64.3)$ & 0.003 \\
\hline Education, median (IQR), years & $4(2-8)$ & $0(0-0)$ & $4(3-4)$ & $7.5(4-11)$ & $<0.001$ \\
\hline Economic level (BECC score), mean (SD) & $19.9(6.2)$ & $16.4(5.8)$ & $19.2(5.5)$ & $21.7(6.5)$ & 0.006 \\
\hline Manual occupation, No. (\%) & 67 (51.9) & $14(93.3)$ & $30(51.7)$ & $23(41.1)$ & 0.001 \\
\hline Married, No. (\%) & $42(32.6)$ & $1(6.7)$ & 19 (32.8) & 22 (39.3) & 0.057 \\
\hline Assistance with medications, No. (\%) & $22(17.1)$ & $5(33.3)$ & $10(17.2)$ & $7(12.5)$ & 0.200 \\
\hline Diabetes knowledge (SKILLD), mean (SD) & $4.2(2.6)$ & $2.5(2.6)$ & $3.5(2.2)$ & $5.3(2.6)$ & $<0.001$ \\
\hline Health literacy (SAHLPA-18), median (IQR) & $13(10-16)$ & $0(0-0)$ & $11.5(10-13)$ & $16(15-17)$ & $<0.001$ \\
\hline Depressive symptoms (GDS-15), median (IQR) & $3(1-5)$ & $4(2.3-5.8)$ & $3(1-5)$ & $2(1-4.5)$ & 0.170 \\
\hline Insulin containing regimen, No. (\%) & 41 (31.8) & $6(40.0)$ & $18(31.0)$ & $17(30.4)$ & 0.772 \\
\hline Diabetes duration, median (IQR), years & $10(5-20)$ & $20(7.3-20)$ & $10(5-19)$ & $12(4.5-20)$ & 0.365 \\
\hline HbA1c, mean (SD) & $7.2(1.4)$ & $6.8(1.1)$ & $7.6(1.6)$ & $7.0(1.1)$ & 0.034 \\
\hline LDL cholesterol, mean (SD) & $110.5(36.5)$ & $105.7(29.6)$ & $108.3(38.5)$ & $114.1(36.3)$ & 0.605 \\
\hline Systolic blood pressure, mean (SD) & $143.1(23.5)$ & $147.3(23.8)$ & $142.9(23.7)$ & $142.0(23.6)$ & 0.741 \\
\hline Diastolic blood pressure, mean (SD) & $79.4(10.8)$ & $78.0(12.1)$ & $79.7(7.9)$ & $79.5(13.1)$ & 0.866 \\
\hline Any microvascular complication, No. (\%) & $33(25.6)$ & $3(20.0)$ & $18(31.0)$ & $12(21.4)$ & 0.468 \\
\hline Retinopathy, №. (\%) & $16(12.4)$ & $0(0)$ & $10(17.2)$ & $6(10.7)$ & 0.210 \\
\hline Nephropathy, No. (\%) & $17(13.2)$ & $1(6.7)$ & $9(15.5)$ & 7 (12.5) & 0.813 \\
\hline Neuropathy, No. (\%) & $15(11.6)$ & $3(20.0)$ & $6(10.3)$ & $6(10.7)$ & 0.538 \\
\hline Any macrovascular complication, No. (\%) & $37(28.7)$ & $5(33.3)$ & $20(34.5)$ & $12(21.4)$ & 0.262 \\
\hline Cerebrovascular disease, No. (\%) & $15(11.6)$ & $0(0)$ & $10(17.2)$ & $5(8.9)$ & 0.142 \\
\hline Coronary artery disease, No. (\%) & $17(13.2)$ & $4(26.7)$ & $9(15.5)$ & $4(7.1)$ & 0.092 \\
\hline Peripheral artery disease, No. (\%) & $11(8.5)$ & $2(13.3)$ & $6(10.3)$ & $3(5.4)$ & 0.451 \\
\hline
\end{tabular}

benefit. ${ }^{26}$ According to that rationale, the influence of health literacy on health outcomes would be stronger at the lower end of the health literacy spectrum, with the association curve tending to reach a plateau at the higher end. Our study, as well as that of Tang et al, ${ }^{10}$ has included a sample which represents properly the lowest levels of the health literacy spectrum, where the association of health literacy with health outcomes is deemed to be stronger. This may explain, at least in part, the convincing associations that have been found in both studies, but more studies with very low educated populations from developing countries are needed to confirm that hypothesis.

In addition to the preceding hypothesis, two additional factors can be invoked to explain the significant association observed in our study between functional health literacy and glycaemic control. First, we have made a careful selection of the sample, excluding conditions that can influence scores in functional health literacy tests, affect the accuracy of the HbA1c assay or determine different targets of glycaemic control. Second, the study was conducted in a governmentfinanced health system which provides medications at no cost. This factor may attenuate inequalities in access to therapeutic resources, making the role of functional health literacy more evident.

After conducting a systematic review, Al Sayah et a 9 suggested that a confounder could explain the inconsistency in results across studies designed to investigate the effects of health literacy on diabetes outcomes. Significant associations between functional health literacy and HbAlc were found mostly in studies that did not adjust for diabetes knowledge. Our study brings a new piece of evidence that is contrary to that hypothesis-we have controlled for diabetes knowledge and have still found a significant association between functional health literacy and glycaemic control. The use of a verbally administered test to evaluate diabetes knowledge in our study may explain this contrasting result. In prior studies, tests of diabetes knowledge which involve reading and writing may have provided measures that are highly correlated with functional health literacy tests, thus suppressing some of the effects of this variable.

In our study, the diabetes knowledge test was moderately correlated with the measure of functional health literacy $(\mathrm{r}=0.39)$ and its corresponding VIF was 1.70, indicating that it did not cause multicollinearity. When SKILLD was added to a model already containing 
Table 2 Association between $\mathrm{HbA1c}$ and patient characteristics in hierarchical multiple linear regression models $(\mathrm{n}=114)$

\begin{tabular}{|c|c|c|c|c|}
\hline \multirow[b]{2}{*}{ Independent variables } & \multicolumn{4}{|c|}{ Standardised $\beta$ coefficients } \\
\hline & $\begin{array}{l}\text { Step 1 } \\
R^{2}=0.06\end{array}$ & $\begin{array}{l}\text { Step } 2 \\
R^{2}=0.15\end{array}$ & $\begin{array}{l}\text { Step } 3 \\
R^{2}=0.29\end{array}$ & $\begin{array}{l}\text { Step } 4 \\
R^{2}=0.29 \\
\end{array}$ \\
\hline Health literacy, SAHLPA-18 score & $-0.25^{\star \star}$ & $-0.31^{* *}$ & $-0.41^{\star \star \star}$ & $-0.42^{\star \star \star}$ \\
\hline Age (years) & & -0.16 & -0.16 & -0.16 \\
\hline Gender (female vs male) & & 0.08 & 0.16 & 0.16 \\
\hline Race (white vs non-white) & & 0.03 & 0.03 & 0.03 \\
\hline Education (years) & & 0.11 & 0.12 & 0.10 \\
\hline Occupation (manual vs non-manual) & & 0.14 & 0.11 & 0.10 \\
\hline Economic status (BECC score) & & 0.16 & 0.15 & 0.15 \\
\hline Marital status (married vs unmarried) & & -0.09 & -0.08 & -0.08 \\
\hline Depressive symptoms, GDS-15 score & & & -0.07 & -0.06 \\
\hline Diabetes duration (years) & & & $0.25^{\star \star}$ & $0.24^{*}$ \\
\hline Insulin containing regimen (yes vs no) & & & 0.17 & 0.16 \\
\hline Assistance with medications (yes vs no) & & & $-0.22^{*}$ & $-0.23^{*}$ \\
\hline Diabetes knowledge, SKILLD score & & & & 0.05 \\
\hline \multicolumn{5}{|c|}{$\begin{array}{l}\text { Multiple linear regression models with HbA1c as the dependent variable, SAHLPA-18 as the primary independent variable and other } \\
\text { characteristics as covariates. The coefficient of determination }\left(\mathrm{R}^{2}\right) \text { indicates the proportion of the variance of } \mathrm{HbA} 1 \mathrm{c} \text { which can be explained } \\
\text { by the set of predictors. } \\
{ }^{*} \mathrm{p}<0.05 \text {. } \\
{ }^{* \star} \mathrm{p}<0.01 \text {. } \\
{ }^{* \star \star} \mathrm{p}<0.001 \text {. } \\
\text { BECC, Brazilian Economic Classification Criterion; GDS-15, } 15 \text {-Item Geriatric Depression Scale; HbA1c, glycosylated haemoglobin; } \\
\text { SAHLPA-18, } 18 \text {-item Short Assessment of Health Literacy for Portuguese-speaking Adults; SKILLD, Spoken Knowledge in Low Literacy } \\
\text { patients with Diabetes. }\end{array}$} \\
\hline
\end{tabular}

SAHLPA-18, demographic characteristics and clinical factors, it was not significantly associated with glycaemic control; it did not change the results and also did not improve the predictive power of the regression model (table 2). Findings from a recent study conducted by Jeppesen $e t a l^{27}$ have raised concerns about the properties of SKILLD. Besides presenting only a moderate correlation with a measure of criterion validity, the test has been shown to have limited inter-rater reliability and low internal consistency. Taking into account the findings of Jeppesen et al and those of the present study, it is worth suggesting that the properties of SKILLD should be carefully investigated in future studies before it can be assumed to provide valid and useful measures.

Contrary to our expectations, illiterate patients did not have poorer glycaemic control when compared to patients with adequate functional health literacy. This finding raises questions on how illiterate patients may compensate for their difficulties. We can speculate that, when caring for patients who report being unable to read at all, health professionals and family members are more aware of the need for compensation strategies. In contrast, among individuals who report being able to read, inadequate health literacy is a frequently unrecognised

Table3 Functional health literacy and poor diabetes outcomes $(n=129)$

\begin{tabular}{|c|c|c|c|c|}
\hline \multirow[b]{2}{*}{ Outcome } & \multirow[b]{2}{*}{ Health literacy level } & \multirow[b]{2}{*}{ No. (\%) } & \multicolumn{2}{|l|}{ OR $(95 \% \mathrm{Cl})$} \\
\hline & & & Unadjusted & Adjusted* $^{*}$ \\
\hline \multirow[t]{3}{*}{ Inadequate glycaemic control ( $\mathrm{HbA} 1 \mathrm{C}>8 \%)$} & Adequate & $9(16.1)$ & 1.00 & 1.00 \\
\hline & Inadequate & $18(31.0)$ & $2.35(0.95$ to 5.81$)$ & $4.76(1.36$ to 16.63$)$ \\
\hline & Illiteracy & $2(13.3)$ & $0.80(0.15$ to 4.19$)$ & $1.17(0.13$ to 10.87$)$ \\
\hline \multirow[t]{3}{*}{ Retinopathy } & Adequate & $6(10.7)$ & 1.00 & 1.00 \\
\hline & Inadequate & $10(17.2)$ & $1.74(0.59$ to 5.15$)$ & $2.88(0.60$ to 13.86$)$ \\
\hline & Illiteracy & $0(0.0)$ & NA & NA \\
\hline \multirow{3}{*}{ Nephropathy } & Adequate & $7(12.5)$ & 1.00 & 1.00 \\
\hline & Inadequate & $9(15.5)$ & $1.29(0.44$ to 3.73$)$ & $0.91(0.19$ to 4.42$)$ \\
\hline & Illiteracy & $1(6.7)$ & 0.50 (0.06 to 4.41$)$ & $0.23(0.01$ to 3.85$)$ \\
\hline \multirow[t]{3}{*}{ Neuropathy } & Adequate & $6(10.7)$ & 1.00 & 1.00 \\
\hline & Inadequate & $6(10.3)$ & 0.96 (0.29 to 3.18$)$ & $0.98(0.22$ to 4.36$)$ \\
\hline & Illiteracy & $3(20.0)$ & $2.08(0.45$ to 9.55$)$ & $1.24(0.15$ to 10.27$)$ \\
\hline
\end{tabular}


condition. ${ }^{5}$ Unfortunately, our study did not include a specific instrument to assess social support, which would have allowed us to explore a possible interaction of this factor with functional health literacy in determining diabetes outcomes. In future studies, instruments designed to assess social support may possibly explain how illiterate patients can compensate for their difficulties.

In an Iranian diabetes clinic, Jahanlou and Karami ${ }^{28}$ did not find a significant difference in HbAlc levels between illiterate $(n=108)$ and literate $(n=148)$ patients. Similarly, Hawthorne and Tomlinson ${ }^{29}$ reported comparable levels of HbAlc between illiterate $(n=54)$ and literate $(n=158)$ Pakistani patients with type 2 diabetes. However, both studies have based their reports on bivariate analyses, without appropriate control for confounding variables. In our study, the relatively small subsample of illiterate patients provided limited power to reject differences with small or even moderate magnitude. Therefore, these preliminary findings regarding glycaemic control in illiterate patients should be confirmed in future studies with adequate sample size and adjustment for confounding variables.

Our study has a number of limitations. First, its crosssectional design does not allow the establishment of causal associations between inadequate functional health literacy and poor diabetes outcomes. Second, although we have excluded individuals with a diagnosis of dementia, we did not screen for dementia and did not make adjustments for cognitive performance. Third, our study was clearly underpowered to investigate the association between functional health literacy and diabetes complications, which presented low prevalence in our sample, varying from $11.6 \%$ (neuropathy) to $13.2 \%$ (nephropathy). Fourth, although SAHLPA-18 has been shown to be valid and to present good psychometric properties in Brazilian older adults, ${ }^{22}$ it does not include tasks to assess some important aspects of health literacy, such as numeracy skills, interactive skills and critical skills. ${ }^{30}$

In conclusion, this study found that, in a sample of low-educated older patients with type 2 diabetes, lower functional health literacy skills were associated with higher odds of poor glycaemic control. These findings reinforce the importance of addressing limited functional health literacy in clinical practice.

Contributors JGS participated in the study design, collected the data and wrote the first draft of the manuscript. DA conceived the study, conducted the statistical analysis and wrote the final draft of the manuscript. RMM, ALB, FC and WJ-F participated in the study design and revised the manuscript

Funding This research received no specific grant from any funding agency in the public, commercial or not-for-profit sectors

Competing interests None.

Ethics approval Comissão de Ética para Análise de Projetos de Pesquisa do Hospital das Clínicas da Faculdade de Medicina da Universidade de São Paulo (CAPPesq)

Provenance and peer review Not commissioned; externally peer reviewed.

Data sharing statement No additional data are available.
Open Access This is an Open Access article distributed in accordance with the Creative Commons Attribution Non Commercial (CC BY-NC 3.0) license, which permits others to distribute, remix, adapt, build upon this work noncommercially, and license their derivative works on different terms, provided the original work is properly cited and the use is non-commercial. See: http:// creativecommons.org/licenses/by-nc/3.0/

\section{REFERENCES}

1. Nutbeam D. Health promotion glossary. Health Promot Int 1998;13:349-64.

2. Parker RM, Baker DW, Williams MV et al. The test of functional health literacy in adults: a new instrument for measuring patients literacy skills. J Gen Intern Med 1995;10:537-41.

3. Berkman ND, Sheridan SL, Donahue KE, et al. Low health literacy and health outcomes: an updated systematic review. Ann Intern Med 2011;155:97-107.

4. Sheridan SL, Halpern DJ, Viera AJ, et al. Interventions for individuals with low health literacy: a systematic review. J Health Commun 2011;16:30-54.

5. Kelly PA, Haidet P. Physician overestimation of patient literacy: a potential source of health care disparities. Patient Educ Couns 2007;66:119-22.

6. Gazmararian JA, Baker DW, Williams MV, et al. Health literacy among Medicare enrollees in a managed care organization. JAMA 1999;281:545-51.

7. Baker DW, Gazmararian JA, Sudano J, et al. The association between age and health literacy among elderly persons. J Gerontol B Psychol Sci Soc Sci 2000;55:S368-74.

8. Paasche-Orlow MK, Wolf MS. The causal pathways linking health literacy to health outcomes. Am J Health Behav 2007;31:S19-26.

9. Al Sayah F, Majumdar SR, Williams B, et al. Health literacy and health outcomes in diabetes: a systematic review. J Gen Intern Med 2013;28:444-52.

10. Tang $\mathrm{YH}$, Pang SM, Chan MF, et al. Health literacy, complication awareness, and diabetic control in patients with type 2 diabetes mellitus. J Adv Nurs 2008;62:74-83.

11. Oliveira MO, Porto CS, Brucki SMD, et al. S-TOFHLA in mild Alzheimer's disease and mild cognitive impairment patients as a measure of functional literacy: preliminary study. Dement Neuropsychol 2009;3:291-8.

12. Sela-Katz P, Rabinowitz I, Shugaev I, et al. Basic knowledge of the medication regimen correlates with performance on cognitive function tests and diagnosis of dementia in elderly patients referred to a geriatric assessment unit. Gerontology 2010;56:491-5.

13. Kim MK, Kwon HS, Baek KH, et al. Effects of thyroid hormone on $\mathrm{A} 1 \mathrm{C}$ and glycated albumin levels in nondiabetic subjects with overt hypothyroidism. Diabetes Care 2010;33:2546-8.

14. Ford ES, Cowie CC, Li C, et al. Iron-deficiency anemia, non-iron-deficiency anemia and $\mathrm{HbA} 1 \mathrm{c}$ among adults in the US. J Diabetes 2011;3:67-73.

15. Sharif $A$, Baboolal K. Diagnostic application of the $A(1 \mathrm{c})$ assay in renal disease. J Am Soc Nephrol 2010;21:383-5.

16. American Geriatrics Society Expert Panel on the Care of Older Adults with Diabetes Mellitus. Guidelines abstracted from the American Geriatrics Society guidelines for improving the care of older adults with diabetes mellitus: 2013 update. J Am Geriatr Soc 2013;61:2020-6.

17. Ensrud KE, Ewing SK, Taylor BC, et al. Comparison of 2 frailty indexes for prediction of falls, disability, fractures, and death in older women. Arch Intern Med 2008;168:382-9.

18. Brazilian Association of Research Companies. Brazilian economic classification criterion. 2008. http://www.abep.org (accessed 5 Dec 2013).

19. Papelbaum M, Moreira RO, Coutinho W, et al. Depression, glycemic control and type 2 diabetes. Diabetol Metab Syndr 2011;3:26.

20. Yesavage JA, Brink TL, Rose TL, et al. Development and validation of a geriatric depression screening scale: a preliminary report. J Psychiatr Res 1982;17:37-49.

21. Almeida OP, Almeida SA. Short versions of the geriatric depression scale: a study of their validity for the diagnosis of a major depressive episode according to ICD-10 and DSM-IV. Int J Geriatr Psychiatry 1999;14:858-65.

22. Apolinario D, Braga RC, Magaldi RM, et al. Short assessment of health literacy for Portuguese-speaking adults. Rev Saude Publica 2012;46:702-11.

23. Rothman RL, Malone $\mathrm{R}$, Bryant $\mathrm{B}$, et al. The spoken knowledge in low literacy in diabetes scale: a diabetes knowledge scale for vulnerable patients. Diabetes Educ 2005;31:215-24. 
24. Faul F, Erdfelder E, Lang AG, et al. G*Power 3: a flexible statistical power analysis program for the social, behavioral, and biomedical sciences. Behav Res Methods 2007;39:175-91.

25. Durlak JA. How to select, calculate, and interpret effect sizes. J Pediatr Psychol 2009;34:917-28.

26. Wolf MS, Feinglass J, Thompson J, et al. In search of 'low health literacy': threshold vs. gradient effect of literacy on health status and mortality. Soc Sci Med 2010;70:1335-41.

27. Jeppesen KM, Hull BP, Raines M, et al. A validation study of the spoken knowledge in low literacy in diabetes scale (SKILLD). J Gen Intern Med 2012;27:207-12.
28. Jahanlou AS, Karami NA. The effect of literacy level on health related-quality of life, self-efficacy and self-management behaviors in diabetic patients. Acta Med Iran 2011;49:153-8.

29. Hawthorne K, Tomlinson S. Pakistani moslems with type 2 diabetes mellitus: effect of sex, literacy skills, known diabetic complications and place of care on diabetes knowledge, reported self-monitoring management and glycaemic control. Diabet Med 1999;16:591-7.

30. Apolinario D, Mansur LL, Carthery-Goulart MT, et al. Detecting limited health literacy in Brazil: development of a multidimensional screening tool. Health Promot Int. Published Online First: 30 October 2013. doi: $10.1093 /$ heapro/dat074 